\title{
Trends and variations in mantle cell lymphoma incidence from 1995 to 2013: A comparative study between Texas and National SEER areas
}

\author{
Shuangshuang Fu' ${ }^{1}$, Michael Wang ${ }^{2}$, David R. Lairson ${ }^{3}$, Ruosha Li $^{4}$, Bo Zhao ${ }^{5}$ and \\ Xianglin L. Du ${ }^{1}$ \\ ${ }^{1}$ Department of Epidemiology, Human Genetics, and Environmental Science, School of Public Health, The University of Texas \\ Health Science Center in Houston, Houston, Texas 77030, USA \\ ${ }^{2}$ Department of Lymphoma and Myeloma, The University of Texas MD Anderson Cancer Center, Houston, Texas 77030, USA \\ ${ }^{3}$ Department of Management Policy and Community Health, School of Public Health, The University of Texas Health Science \\ Center in Houston, Houston, Texas 77030, USA \\ ${ }^{4}$ Department of Biostatistics, School of Public Health, The University of Texas Health Science Center in Houston, Houston, \\ Texas 77030, USA \\ ${ }^{5}$ Department of Medicine, Baylor College of Medicine, Houston, Texas 77030, USA \\ Correspondence to: Xianglin L. Du, email: Xianglin.L.Du@uth.tmc.edu \\ Michael Wang, email: miwang@mdanderson.org
}

Keywords: mantle cell lymphoma, incidence, SEER, Texas, disparity

Received: June 10, 2017 Accepted: October 02, $2017 \quad$ Published: November 03, 2017

Copyright: Fu et al. This is an open-access article distributed under the terms of the Creative Commons Attribution License 3.0 (CC BY 3.0), which permits unrestricted use, distribution, and reproduction in any medium, provided the original author and source are credited.

\section{ABSTRACT}

Background: Few studies have assessed mantle cell lymphoma (MCL) incidence trends in the U.S. National Cancer Institute's Surveillance, Epidemiology and End Results (SEER) areas. Previous studies were 5 to 9 years old and MCL incidence in Texas remains unknown. This study updated the temporal trends and variations of MCL incidence in the SEER areas and compared them with counterpart data in Texas.

Results: From 1995 to 2013, there were 2, 435 and 5, 193 newly diagnosed MCL patients in Texas and SEER areas. Age-adjusted MCL incidence was 0.91 per 100,000 persons per year in Texas and 1.01 in SEER areas. MCL incidence increased steadily with an annual percent change (APC) of $2.56 \%$ in SEER areas and an APC of $2.16 \%$ in Texas. In SEER areas, APCs for MCL incidence were significantly different from zero in patients with advanced stage tumor $(3.33 \%)$, male $(2.71 \%)$, elderly patients $\geq 80$ years old $(4.21 \%)$ and non-Hispanic white patients $(2.83 \%)$ (all $P<0.05)$. Similar patterns were found in Texas for both incidence rates and APCs.

Materials and methods: We identified all adult patients with newly diagnosed MCL in Texas Cancer Registry and SEER databases from 1995 to 2013. Age-adjusted incidence rates were calculated and negative binomial regression model was used to assess the factors associated with MCL incidence.

Conclusions: MCL incidence rates increased over time in both Texas and SEER areas, with increases being greater in male, non-Hispanic white, and elderly patient $\geq 70$ years with advanced stage tumors. Texas has similar MCL incidence trends and disparities as the national SEER areas.

\section{INTRODUCTION}

Non-Hodgkin lymphoma (NHL) is the seventh most common cancer and eighth leading cause of cancer death in the U.S. [1]. NHL is also a heterogeneous group of lymphomas; each subtype of NHL has different prognosis and treatment options [2]. Mantle cell lymphoma (MCL) is a rare, aggressive subtype of B-cell NHL, affecting 3\% to $6 \%$ of patients with NHL $[3,4]$. MCL has a poor prognosis, with a median overall survival of 4 to 5 years [5]. Previous studies conducted in the U.S. National Cancer Institute's Surveillance, 
Epidemiology and End Results (SEER) areas showed that MCL incidence has increased steadily from 1992 $[3,6]$ when it was established as a distinct type of lymphoma [7].

Previous studies $[3,6]$ also showed that both prevalence and incidence of MCL were higher in older, male and white populations in the U.S. SEER areas. Only a few studies have been conducted to determine the incidence trends and variations in MCL incidence in the U.S. SEER areas [3, 6, 8], but relatively little is known about its incidence by sociodemographic characteristics and tumor stage. Texas is the second most populated state in the U.S., but is not included in the U.S. SEER registries. In Texas, MCL incidence and disease characteristics remain unexplored and the data were not released until 1995. In SEER areas, the previous MCL incidence studies extended only to 2009 , but new data is available, so a new analysis is needed.

Therefore, the purpose of the present study is twofold. First, we updated the temporal trends and variations of MCL incidence in the U.S. SEER areas and compared them with counterpart data in Texas from 1995 to 2013. Second, we evaluated the variations in MCL incidence by age, gender, race/ethnicity and tumor stage. The present study provides a more comprehensive overview of the MCL incidence and factors associated with the development of MCL at the state and national level, which is important information for unfolding the etiology of MCL and identifying high risk population for disease prevention.

\section{RESULTS}

\section{Patient characteristics}

From 1995 to 2013, there were 2,435 and 5,193 newly diagnosed MCL cases in Texas and SEER areas, accounting for $3.48 \%$ and $3.65 \%$ of patients with NHL, respectively. The median age at diagnosis was 68 years in Texas and 69 years in SEER areas. Table 1 presents the total number of MCL patients in Texas and SEER, and demographic characteristics for those patients. In Texas, $71.17 \%$ of the MCL patients were male, $78.85 \%$ were non-Hispanic white, and $61.15 \%$ had advanced stage tumor. In SEER areas, 68.75\% were male, $80.53 \%$ were non-Hispanic white, and $75.47 \%$ had advanced stage tumor. The percentage of MCL patients with advanced stage tumor in SEER areas was $14.32 \%$ higher than that in Texas.

\section{MCL incidence rate and relative risk (RR) Over 19 years in Texas and SEER areas}

Table 2 presents MCL incidence rates and RRs in Texas and SEER. From 1995 to 2013, overall ageadjusted MCL incidence was 0.91 per 100,000 persons per year in Texas and 1.01 per 100,000 persons per year in SEER areas. In Texas, the risk of MCL increased with age and was highest in patients aged $\geq 70$ years when compared to those aged under 50 (RR for $70-$ 79 years: $32.27,95 \% \mathrm{CI}$ : 27.30-38.15; RR for $80+$ years: $32.04,95 \%$ CI: 26.69-38.47). The risk of MCL was higher in male (RR: 3.00, 95\% CI: 2.72-3.30) compared to females. Both non-Hispanic white (RR: 2.47, 95\% CI: 2.03-3.01) and Hispanic (RR: 1.42, 95\% CI: 1.15-1.77) had a higher risk of developing MCL compared to non-Hispanic Black. The incidence rate for patients with advanced stage tumor was about five times that of localized stage (RR: 4.91, 95\% CI: 4.30-5.59). In SEER areas, the risk of MCL increased with age and was highest in patients aged $\geq 80$ years (RR: 42.01, 95\% CI: 35.75-49.36). The risk of MCL was also higher in male (RR: 2.65, 95\% CI: 2.43-2.89), non-Hispanic white (RR: 2.24, 95\% CI: 1.92-2.61), and patients with advanced stage tumor (RR: $6.88,95 \% \mathrm{CI}$ : 6.11-7.73).

\section{MCL incidence trend over 19 years by demographic factors}

Figure 1 shows that overall MCL incidence increased steadily in SEER areas with an annual percent change (APC) of 2.56\% $(p<0.05)$, but in Texas, the incidence increased from 1995 to 2006, then became stable from 2007 to 2013, with an APC of $2.16 \%(p<0.05)$. Figure 2 shows that in both SEER and Texas, MCL incidence rates for patients with advanced stage tumors increased significantly (APC for SEER: $3.33 \%$ vs. Texas: $3.40 \%$, all $p<0.05$ ). MCL incidence increase was steeper from 1995 to 2005 and then plateaued after 2007. Figure 3 presents MCL incidence trends by gender. In SEER areas, MCL incidence rates experienced a greater increase for male (APC 2.71\%,p<0.05) compared to female (APC $1.99 \%, p<0.05)$ over the past two decades. However, in Texas, MCL incidence rates in female (APC 2.22\%, $p<0.05$ ) increased more compared to male (APC $2.02 \%, p<0.05)$. Figure 4 presents MCL incidence rates by age group. In SEER areas, three age groups had significant increases in MCL incidence; patients aged 60-69 years had an APC of 1.74\% $(p<0.05)$, patients aged $70-79$ had an APC of $3.29 \%(p<0.05)$, and patients aged $\geq 80$ years had an APC of $4.21 \%$ $(p<0.05)$. In Texas, only patients aged $70-79$ years had a significant increase in MCL incidence with an APC of $3.62 \%(p<0.05)$. Figure 5 presents MCL incidence rates by race/ethnicity. MCL incidence rates increased significantly in non-Hispanic white and Hispanic population for both SEER areas (nonHispanic white APC: $2.83 \%$, Hispanic APC: $3.50 \%$, all $p<0.05$ ) and Texas (non-Hispanic white APC: $2.53 \%$, Hispanic: $2.50 \%$, all $p<0.05)$. 
Table 1: Demographic characteristics of MCL patients in Texas and SEER areas, 1995-2013

\begin{tabular}{|c|c|c|c|c|}
\hline \multirow{2}{*}{ Year of diagnosis } & \multicolumn{4}{|c|}{ Number and column \% of cancer cases } \\
\hline & \multicolumn{2}{|c|}{ Texas } & \multicolumn{2}{|c|}{ SEER } \\
\hline 1995-1999 & 405 & 16.63 & 949 & 18.27 \\
\hline $2000-2004$ & 463 & 19.01 & 960 & 18.49 \\
\hline $2005-2008$ & 726 & 29.82 & 1,510 & 29.08 \\
\hline 2009-2013 & 841 & 34.54 & 1,774 & 34.16 \\
\hline \multicolumn{5}{|l|}{ Age category } \\
\hline$<50$ & 200 & 8.21 & 368 & 7.09 \\
\hline $50-59$ & 428 & 17.58 & 932 & 17.95 \\
\hline $60-69$ & 736 & 30.23 & 1,399 & 26.94 \\
\hline $70-79$ & 704 & 28.91 & 1,498 & 28.85 \\
\hline$\geq 80$ & 367 & 15.07 & 996 & 19.18 \\
\hline \multicolumn{5}{|l|}{ Gender } \\
\hline Male & 1,733 & 71.17 & 3,570 & 68.75 \\
\hline Female & 702 & 28.83 & 1,623 & 31.25 \\
\hline \multicolumn{5}{|l|}{ Race } \\
\hline Non-Hispanic Black & 108 & 4.44 & 220 & 4.24 \\
\hline Non-Hispanic White & 1,920 & 78.85 & 4,182 & 80.53 \\
\hline Hispanic & 366 & 15.03 & 445 & 8.57 \\
\hline Other & 41 & 1.68 & 346 & 6.66 \\
\hline \multicolumn{5}{|l|}{ Marital Status } \\
\hline Single & 141 & 5.79 & 526 & 10.13 \\
\hline Married (including common law) & 933 & 38.32 & 3,257 & 62.72 \\
\hline Divorced, separated, or widowed & 290 & 11.91 & 1,076 & 20.72 \\
\hline Unknown & 1,071 & 43.98 & 334 & 6.43 \\
\hline \multicolumn{5}{|l|}{ Tumor Stage } \\
\hline Localized & 302 & 12.40 & 546 & 10.51 \\
\hline Regional & 181 & 7.43 & 428 & 8.24 \\
\hline Advanced & 1,489 & 61.15 & 3,919 & 75.47 \\
\hline Unknown & 463 & 19.01 & 300 & 5.78 \\
\hline Total & 2,435 & & 5,193 & \\
\hline
\end{tabular}

Incidence and trend analyses broken down by year of diagnosis and age of diagnosis for non-hispanic white, male and patients with advanced stage tumor

Table 3 presents the subgroup incidence and trend analyses for non-Hispanic white, male and patients with advanced stage tumor. These three groups of patients had higher incidence rates compared to their reference groups and had experienced higher increase in incidence rates. Therefore, subgroup analyses by year of diagnosis and age at diagnosis were conducted for those three groups and compared between
SEER areas and Texas. MCL incidence rate increases in nonHispanic white and male population were higher in SEER areas compared to Texas (non-Hispanic white APC, SEER: $2.83 \%$ vs. Texas: $2.53 \%$; male APC, SEER: $2.71 \%$ vs. Texas: $2.02 \%$, all $p<0.05$ ). However, MCL incidence increase in patients with advanced stage tumor in SEER areas was slightly lower compared to that in Texas (APC in SEER: $3.33 \%$ vs. Texas: 3.40 , all $p<0.05$ ). We also found that in SEER areas, elderly males had the highest MCL incidence rates (IRs) among all subgroups with high MCL incidence (70-79 years, IR: $6.24,95 \%$ CI: $5.86-6.63 ; 80+$ years, IR: $7.22,95 \%$ CI: $6.66-7.81)$, indicating that substantial 
Table 2: Age-adjusted incidence rates and rate ratios of MCL in Texas and SEER areas, 1995-2013

\begin{tabular}{|c|c|c|c|c|}
\hline & \multicolumn{2}{|c|}{ Texas } & \multicolumn{2}{|c|}{ SEER } \\
\hline & Incidence $^{\mathrm{a}}(95 \% \mathrm{CI})$ & $\operatorname{RR}^{\mathrm{b}}(95 \% \mathrm{CI})$ & Incidence $^{\mathrm{a}}(95 \% \mathrm{CI})$ & $\operatorname{RR}^{\mathrm{b}}(95 \% \mathrm{CI})$ \\
\hline \multicolumn{5}{|l|}{ Year of diagnosis } \\
\hline 1995-1999 & $0.70(0.63-0.77)$ & 1.00 (Reference) & $0.80(0.75-0.85)$ & 1.00 (Reference) \\
\hline 2000-2004 & $0.91(0.82-0.99)$ & $1.26(1.08-1.46)$ & $0.94(0.88-1.00)$ & $1.14(1.00-1.30)$ \\
\hline 2005-2008 & $1.01(0.93-1.08)$ & $1.39(1.21-1.60)$ & $1.09(1.03-1.15)$ & $1.26(1.11-1.43)$ \\
\hline 2009-2013 & $1.00(0.93-1.07)$ & $1.39(1.21-1.60)$ & $1.15(1.09-1.20)$ & $1.34(1.19-1.52)$ \\
\hline PC 1995-2013, \% & \multicolumn{2}{|c|}{53.57} & \multicolumn{2}{|c|}{88.42} \\
\hline APC 1995-2013, \% & \multicolumn{2}{|c|}{$2.16^{*}$} & \multicolumn{2}{|c|}{$2.56^{*}$} \\
\hline \multicolumn{5}{|l|}{ Age (years) } \\
\hline$<50$ & $0.11(0.10-0.13)$ & 1.00 (Reference) & $0.11(0.10-0.13)$ & 1.00 (Reference) \\
\hline $50-59$ & $0.89(0.81-0.98)$ & $7.88(6.60-9.40)$ & $1.03(0.97-1.10)$ & $8.49(7.25-9.95)$ \\
\hline $60-69$ & $2.42(2.25-2.60)$ & $20.29(17.17-23.97)$ & $2.48(2.35-2.61)$ & $21.23(18.23-24.73)$ \\
\hline $70-79$ & $3.65(3.38-3.93)$ & $32.27(27.30-38.15)$ & $3.99(3.79-4.20)$ & $36.46(31.31-42.46)$ \\
\hline$\geq 80$ & $3.38(3.04-3.74)$ & $32.04(26.69-38.47)$ & $4.15(3.90-4.42)$ & $42.01(35.75-49.36)$ \\
\hline \multicolumn{5}{|l|}{ Gender } \\
\hline Female & $0.48(0.45-0.52)$ & 1.00 (Reference) & $0.57(0.54-0.59)$ & 1.00 (Reference) \\
\hline Male & $1.46(1.39-1.53)$ & $3.00(2.72-3.30)$ & $1.58(1.52-1.63)$ & $2.65(2.43-2.89)$ \\
\hline \multicolumn{5}{|l|}{ Race } \\
\hline Non-Hispanic Black & $0.42(0.34-0.51)$ & 1.00 (Reference) & $0.49(0.43-0.57)$ & 1.00 (Reference) \\
\hline Non-Hispanic White & $1.08(1.03-1.13)$ & $2.47(2.03-3.01)$ & $1.18(1.15-1.22)$ & $2.24(1.92-2.61)$ \\
\hline Hispanic & $0.65(0.58-0.72)$ & $1.42(1.15-1.77)$ & $0.79(0.72-0.87)$ & $1.42(1.18-1.69)$ \\
\hline Other & $0.51(0.35-0.71)$ & $1.17(0.81-1.68)$ & $0.56(0.50-0.62)$ & $1.07(0.89-1.28)$ \\
\hline \multicolumn{5}{|l|}{ Tumor stage } \\
\hline Localized & $0.11(0.10-0.13)$ & 1.00 (Reference) & $0.11(0.10-0.12)$ & 1.00 (Reference) \\
\hline Regional & $0.07(0.06-0.08)$ & $0.60(0.50-0.72)$ & $0.08(0.08-0.09)$ & $0.76(0.66-0.89)$ \\
\hline Advanced & $0.56(0.53-0.59)$ & $4.91(4.30-5.59)$ & $0.76(0.73-0.78)$ & $6.88(6.11-7.73)$ \\
\hline Unknown & $0.17(0.16-0.19)$ & $1.53(1.31-1.78)$ & $0.06(0.05-0.07)$ & $0.55(0.47-0.65)$ \\
\hline
\end{tabular}

$95 \%$ CI indicates $95 \%$ confidence interval.

ancidence rate was per 100,000 persons per year and were age-adjusted to the U.S. 2000 Census using the SEER*Stat statistical program.

${ }^{b}$ Relative risk was adjusted for year of diagnosis, age, gender, and tumor stage.

*Significantly different from $0, P<0.05$.

Abbreviations. PC, percent change. APC, annual percent change.

disparities in MCL incidence exist in gender and age groups. Texas had similar patterns in incidence rate distribution by demographic characteristics (male 70-79 years, IR: 5.55, 95\% CI: 5.06-6.08; male 80+ years, IR: 6.06, 95\% CI: 5.31-6.89), but the rates were slightly lower compared to SEER areas.

\section{Overall survival (OS) for MCL patients in SEER and TCR areas}

Median OS was 52 months and 57 months for MCL patients in SEER and TCR areas, respectively.
There was no statistically significant difference in the OS between SEER and TCR (Figure 6). Figure 7 presents the cumulative incidence of death by calendar period of diagnosis. The cumulative incidence of death decreased over time, but for patients diagnosed in each time period, there were no significant differences between the two registries. Figure 8 presents the cumulative incidence of death by tumor stage. The cumulative incidence of death was lowest for patients with localized stage tumor and highest for patients with distant stage tumor. Nevertheless, for patients diagnosed with each stage tumor, there was no 
Table 3: MCL incidence rates for non-Hispanic white, male and patients with advanced stage tumor, SEER and Texas areas, 1995-2013

\begin{tabular}{|c|c|c|c|c|c|c|}
\hline \multicolumn{7}{|c|}{ SEER } \\
\hline & \multicolumn{2}{|c|}{ non-Hispanic White } & \multicolumn{2}{|l|}{ Male } & \multicolumn{2}{|c|}{ Advanced stage tumor } \\
\hline & Incidence $^{\mathrm{a}} 95 \% \mathrm{CI}$ & Count & Incidence $^{\mathrm{a}} 95 \% \mathrm{CI}$ & Count & Incidence ${ }^{\mathrm{a}} \% \mathrm{CI}$ & Count \\
\hline \multicolumn{7}{|l|}{ Year of diagnosis } \\
\hline 1995-1999 & $0.88(0.82-0.94)$ & 849 & $1.20(1.11-1.3)$ & 622 & $0.55(0.51-0.59)$ & 653 \\
\hline $2000-2003$ & $1.05(0.98-1.12)$ & 856 & $1.52(1.4-1.64)$ & 679 & $0.70(0.65-0.76)$ & 721 \\
\hline 2004-2008 & $1.25(1.19-1.32)$ & 1,363 & $1.71(1.6-1.81)$ & 1,044 & $0.83(0.78-0.88)$ & 1,157 \\
\hline 2009-2013 & $1.31(1.24-1.37)$ & 1,549 & $1.78(1.68-1.89)$ & 1,225 & $0.89(0.84-0.94)$ & 1,388 \\
\hline 1995-2013 & $1.13(1.1-1.17)$ & 4,617 & $1.58(1.52-1.63)$ & 3,570 & $0.76(0.73-0.78)$ & 3,919 \\
\hline PC 1995-2013 & 99.72 & & 109.82 & & 156.66 & \\
\hline APC 1995-2013 & $2.83^{*}$ & & $2.71^{*}$ & & $3.33^{*}$ & \\
\hline \multicolumn{7}{|l|}{ Age at diagnosis } \\
\hline $20-49$ & $0.14(0.12-0.16)$ & 262 & $0.16(0.14-0.18)$ & 261 & $0.09(0.08-0.1)$ & 289 \\
\hline $50-59$ & $1.25(1.16-1.35)$ & 729 & $1.53(1.42-1.65)$ & 675 & $0.84(0.78-0.91)$ & 762 \\
\hline $60-69$ & $2.87(2.7-3.05)$ & 1,088 & $3.76(3.53-4.01)$ & 1,001 & $1.94(1.83-2.06)$ & 1,098 \\
\hline $70-79$ & $4.69(4.43-4.96)$ & 1,244 & $6.24(5.86-6.63)$ & 1,015 & $2.93(2.76-3.11)$ & 1,100 \\
\hline $80+$ & $4.72(4.41-5.04)$ & 859 & $7.22(6.66-7.81)$ & 618 & $2.80(2.59-3.02)$ & 670 \\
\hline \multicolumn{7}{|c|}{ Texas } \\
\hline & \multicolumn{2}{|c|}{ non-Hispanic White } & \multicolumn{2}{|l|}{ Male } & \multicolumn{2}{|c|}{ Advanced stage tumor } \\
\hline & Incidence $^{\mathrm{a}} 95 \% \mathrm{CI}$ & Count & Incidence $^{\mathrm{a}} 95 \% \mathrm{CI}$ & Count & Incidence $^{\mathrm{a}} 95 \% \mathrm{CI}$ & Count \\
\hline \multicolumn{7}{|l|}{ Year of diagnosis } \\
\hline 1995-1999 & $0.79(0.71-0.88)$ & 326 & $1.12(1.00-1.27)$ & 289 & $0.34(0.29-0.39)$ & 195 \\
\hline $2000-2003$ & $1.07(0.97-1.19)$ & 377 & $1.39(1.24-1.55)$ & 318 & $0.56(0.50-0.63)$ & 288 \\
\hline 2004-2008 & $1.19(1.10-1.30)$ & 574 & $1.66(1.52-1.81)$ & 535 & $0.66(0.60-0.72)$ & 474 \\
\hline 2009-2013 & $1.21(1.11-1.31)$ & 643 & $1.54(1.42-1.68)$ & 591 & $0.63(0.58-0.69)$ & 532 \\
\hline 1995-2013 & $1.08(1.03-1.13)$ & 1,920 & $1.46(1.39-1.53)$ & 1,733 & $0.56(0.53-0.59)$ & 1,489 \\
\hline PC 1995-2013 & 69.42 & & 66.72 & & 144.74 & \\
\hline APC 1995-2013 & $2.53^{*}$ & & $2.02^{*}$ & & $3.40^{*}$ & \\
\hline \multicolumn{7}{|l|}{ Age at diagnosis } \\
\hline $20-49$ & $0.14(0.12-0.17)$ & 136 & $0.17(0.15-0.2)$ & 154 & $0.07(0.06-0.08)$ & 119 \\
\hline $50-59$ & $1.02(0.91-1.15)$ & 301 & $1.37(1.23-1.53)$ & 323 & $0.54(0.48-0.61)$ & 262 \\
\hline $60-69$ & $2.92(2.69-3.17)$ & 591 & $3.84(3.52-4.17)$ & 552 & $1.51(1.38-1.66)$ & 459 \\
\hline $70-79$ & $4.29(3.95-4.66)$ & 583 & $5.55(5.06-6.08)$ & 469 & $2.21(2.01-2.44)$ & 427 \\
\hline $80+$ & $3.80(3.38-4.24)$ & 309 & $6.06(5.31-6.89)$ & 235 & $2.04(1.78-2.33)$ & 222 \\
\hline
\end{tabular}

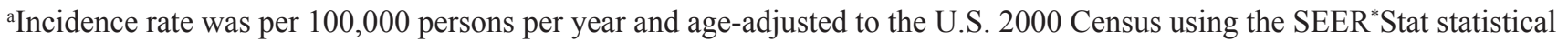
program.

"Significantly different from $0, P<0.05$.

significant difference in the cumulative incidence of death between the two registries.

\section{DISCUSSION}

This study assessed the incidence trends and variations for MCL over the past two decades. It is the first study to compare the results of MCL incidence trends in Texas to those of SEER areas. Patient characteristics by age, gender, race/ethnicity and tumor stage had similar patterns between Texas and SEER areas. MCL incidence disparities by gender and race/ethnicity existed in both Texas and SEER areas. The MCL incidence rates in Texas were slightly lower than that in SEER areas. One 
of the reasons for a lower rate in Texas is a possible under-ascertainment of Texas cases. Another reason is the increasing Texas population with more young people moving to Texas. However, all incidence rates were ageadjusted to the 2000 U.S. standard population to remove potential confounding by age differences.

This study showed that in SEER areas, MCL incidence rates increased steadily and significantly in elderly patients aged 60 years or older. This finding is consistent with previous findings on higher MCL incidence in the elderly population in the U.S. SEER areas [3]. Texas MCL incidence rates increased significantly only in patients aged 70 to 80 years old. The reasons why MCL increased more in the elderly population remain unknown, but elderly people could have more chronic diseases and were more likely to seek medical services, increasing opportunistic findings for MCL [9, 10]. This information is important for informing future drug development and clinical management for MCL patients, since elderly patients are more likely to have multiple chronic complications and suffer from drugrelated side effects and the elderly population tends to be underrepresented in clinical trials [11, 12].

This study also revealed that in SEER areas, the age-adjusted incidence rate for advanced stage MCL was the highest among all tumor stages. The increase of MCL incidence in advanced stage MCL was also stronger than that in any other tumor stages. MCL incidence increased steadily from 1995 to 2006, and then decreased slightly from 2006 to 2008; after 2008, MCL incidence continued to increase steadily. Similar results were observed in Texas, except that Texas had a lower incidence rate in advanced stage tumor and experienced a slight drop in incidence from 2004 to 2013. This increasing trend over the past 20 years can be explained by a few reasons. MCL patients at early stage are usually asymptomatic, therefore, it is difficult to detect MCL at an early stage. In recent years, doctors are more aware of the behavior of MCL and are doing gastrointestinal endoscopy evaluations for stage 1 or 2 patients which were not initially done, some MCL patients were upstaged because of upper/ lower gastrointestinal endoscopies even if the patients were asymptomatic. Also, better tools are available to detect MCL, such as positron emission tomography scanning and peripheral blood markers analyses by flow cytometry. The percentage of advanced stage tumor in SEER areas is $14.32 \%$ higher than that in Texas. The difference is largely due to a larger percent of patients in Texas with unknown tumor stage (Table 1).

MCL was found in predominantly male and nonHispanic white populations in Texas and in SEER areas. The reason why MCL occurred more in male and nonHispanic white is unknown, but studies have shown that men and women are exposed to different occupational hazards $[13,14]$. Genetic studies have shown that $\mathrm{t}(11 ; 14)$ translocation is a molecular hallmark in MCL development [15]. The prevalence of translocation $t(11 ; 14)$ in healthy populations is low, and tends to be slightly higher in males and whites compared to females and blacks [16, 17]. Future studies may address the gender and race/ethnicity disparities in MCL incidence rates by evaluating whether there are disparities in genetic mutation and environmental exposures to toxic agents or radiation by gender and race/ ethnicity because both genetic and environmental factors are highly relevant to the development of tumors.

Our study showed that MCL incidence in the elderly and white population with advanced stage tumor has been increasing. Given the fact that age is an important factor affecting the treatment regimen selection, future studies on therapeutic agents should target more in the elderly patient group. The study showed the decreased mortality

1.4
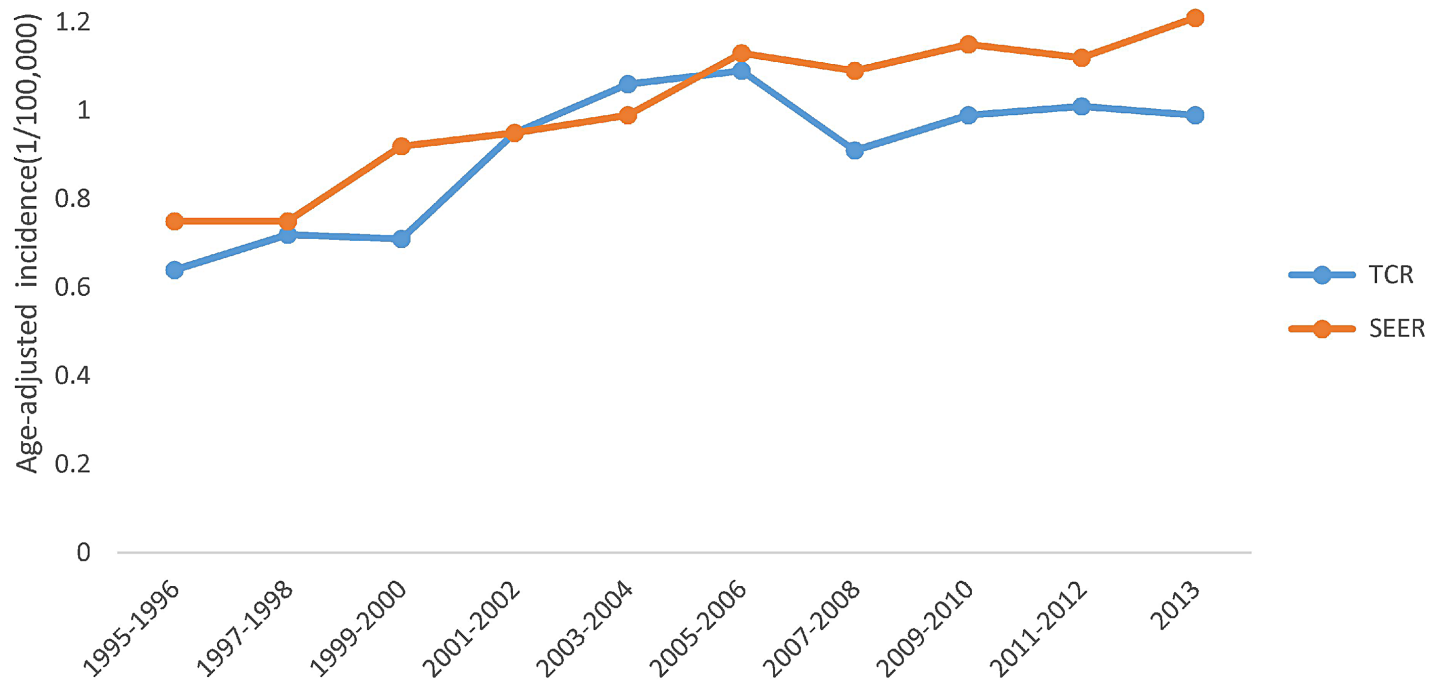

Figure 1: MCL age-adjusted incidence rates over time, 1995-2013. 
rates over time in both SEER and TCR areas, indicating that patients have benefited from the development of novel agents. More detailed analyses on survival trends and the impact of novel agents on the survivorship are warranted.

A series of novel agents have been approved by the FDA to treat MCL since the late 1990s, including rituximab, bortezomib, temsirolimus, bendamustine, lenalidomide, and ibrutinib [18-22]. The treatment regimens for MCL patients differ by age. For symptomatic elderly patients, non-intensive regimens are recommended as first-line treatment regimens, the most common two regimens are rituximab plus cyclophosphamide, doxorubicin, vincristine, and prednisone regimen (R-CHOP) and rituximab plus bendamustine regimen $[5,19]$. For young patients under 65 , aggressive therapy regimens have revealed promising outcomes from several studies and are recommended as first-line treatment, including two major aggressive regimens (Hyper-CVAD regimen and Nordic regimen) [23, 24]. These intensive treatment regimens are mostly recommended for healthier and younger patients because the healthier and younger patients have better tolerance. Therefore, our study findings indicate that in the future, drug development for MCL should focus more on the elderly population.

The study has two important limitations. First, since these two databases only cover around $22 \%$ of the US population, the results may not be generalizable to the entire U.S. population. Second, a number of important known risk factors such as genetics and family history are not available in the data and thus cannot be studied. The differences in these factors may contribute to the observed differences in MCL incidence. The study has important strengths.
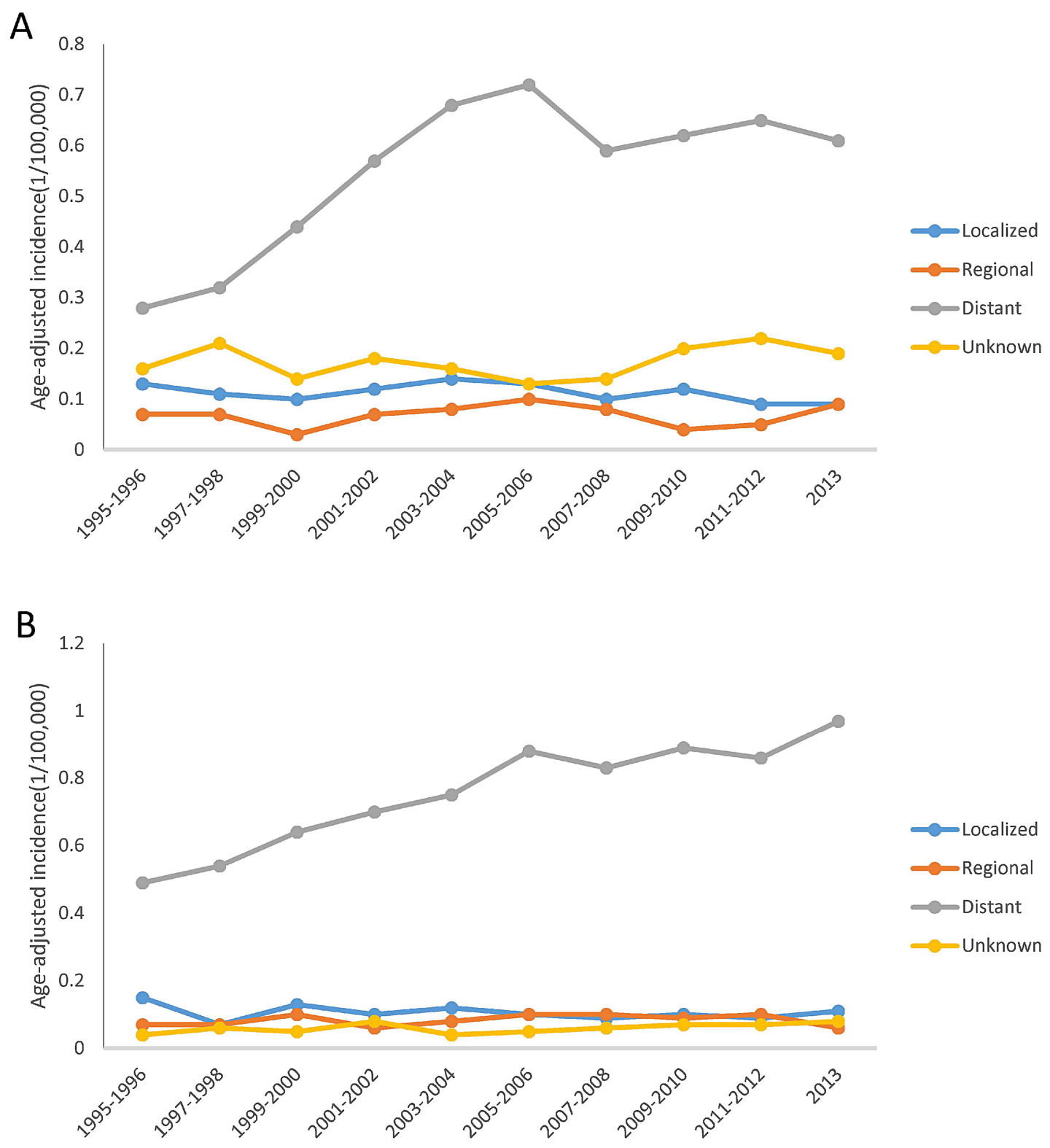

Figure 2: MCL age-adjusted incidence rates over time by tumor stage, 1995-2013. (A) Texas. (B) SEER. 
First, SEER and TCR data include highly valid patient information, tumor variables and survivorship. Second, both TCR and SEER cancer registries have been running for many years, which allow us to generate time trend analysis in the MCL incidences. Adding information from Texas provides a more comprehensive understanding of MCL disease characteristics by different geographic locations.

\section{MATERIALS AND METHODS}

\section{Data sources}

All newly diagnosed MCL cases were obtained through Texas Cancer Registry (TCR) and SEER public-use data from 1995 to 2013 . TCR is a statewide registry measuring Texas cancer burden, diagnosis, treatment and survivorship; it is also one of the largest cancer registries in the United States covering around $8 \%$ of U.S. population [25]. From 1992 to 1999, SEER had 13 registries and covered $14 \%$ of US population [26]. After 2000, SEER registries were expanded to 18 registries (original 13 registries and extra 5 registries included) capturing around $26 \%$ of the U.S. population [26]. Because our study compared the MCL incidence between Texas and SEER from 1995 to 2013, we selected cases in 13 SEER areas to make the population more consistent over time between the two datasets.

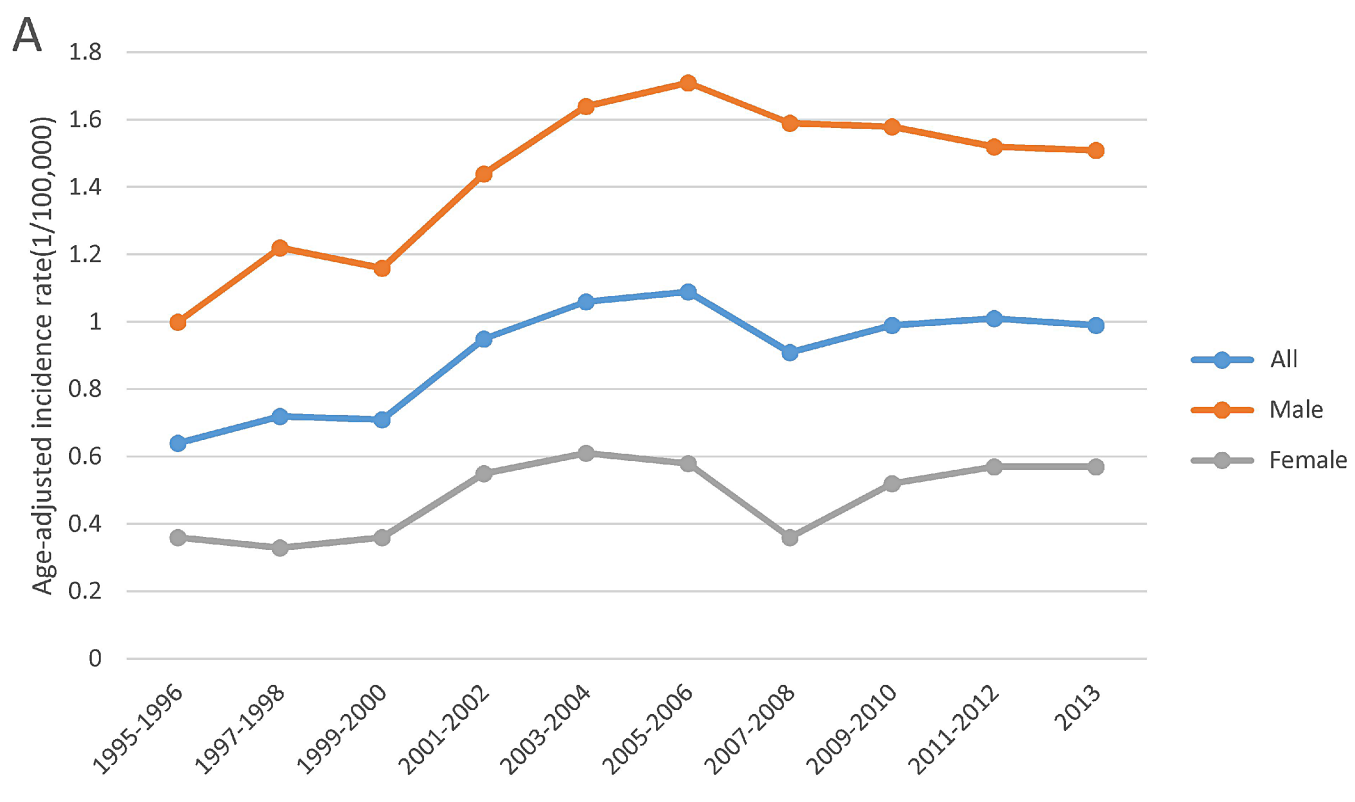

$\mathrm{B}_{2.5}$

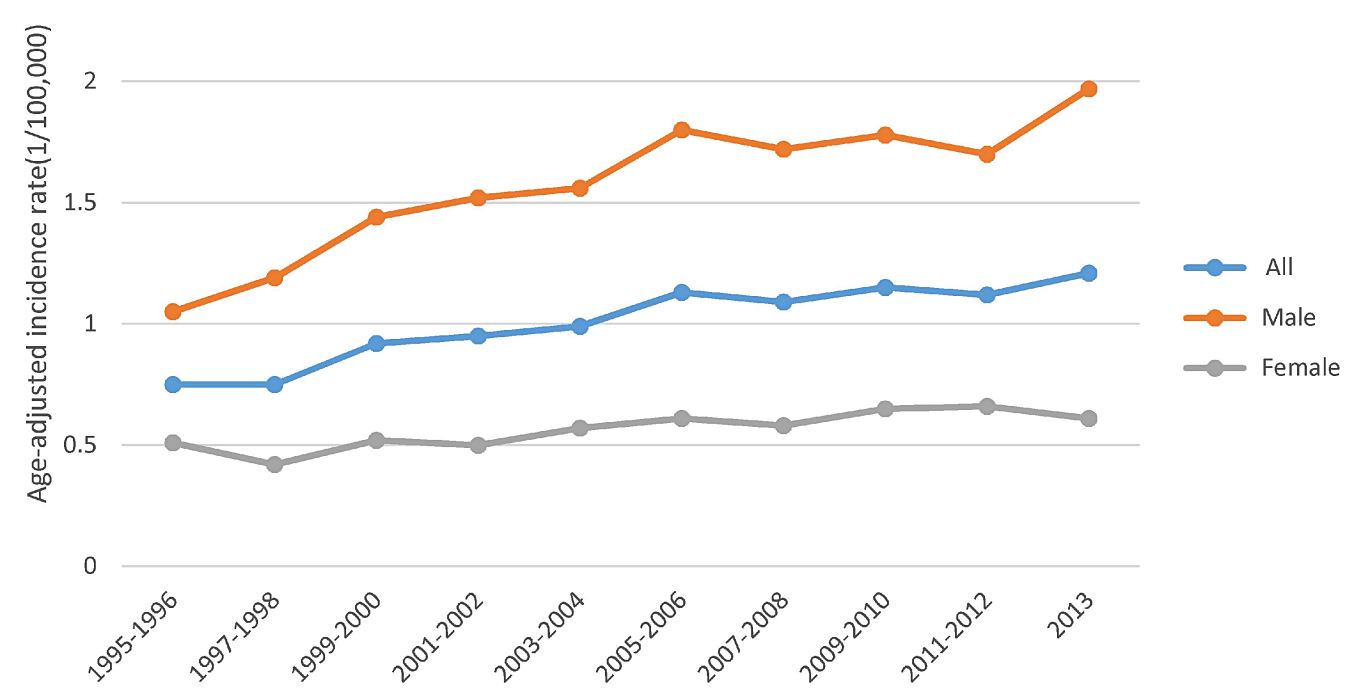

Figure 3: MCL age-adjusted incidence trends by sex, 1995-2013. (A) Texas. (B) SEER. 


\section{Study design and study population}

This is a retrospective cohort study. We identified all newly diagnosed adult MCL patients residing in Texas and in 13 U.S. SEER areas from 1995 to 2013 recorded in TCR and SEER databases. Patients were included if they: (a) had a date of initial MCL diagnosis in 1995 or later; (b) had a primary diagnosis of MCL (International Classification of Diseases for Oncology 3rd edition [ICD-O-3] site code: 9673); and (c) were 20 or older at the time of diagnosis.

\section{Study variables}

\section{Main exposure variables}

The main exposure variables are year of diagnosis defined as four calendar year periods (1995-1999, 2000 2003, 2004-2008, 2009-2013) and geographic areas
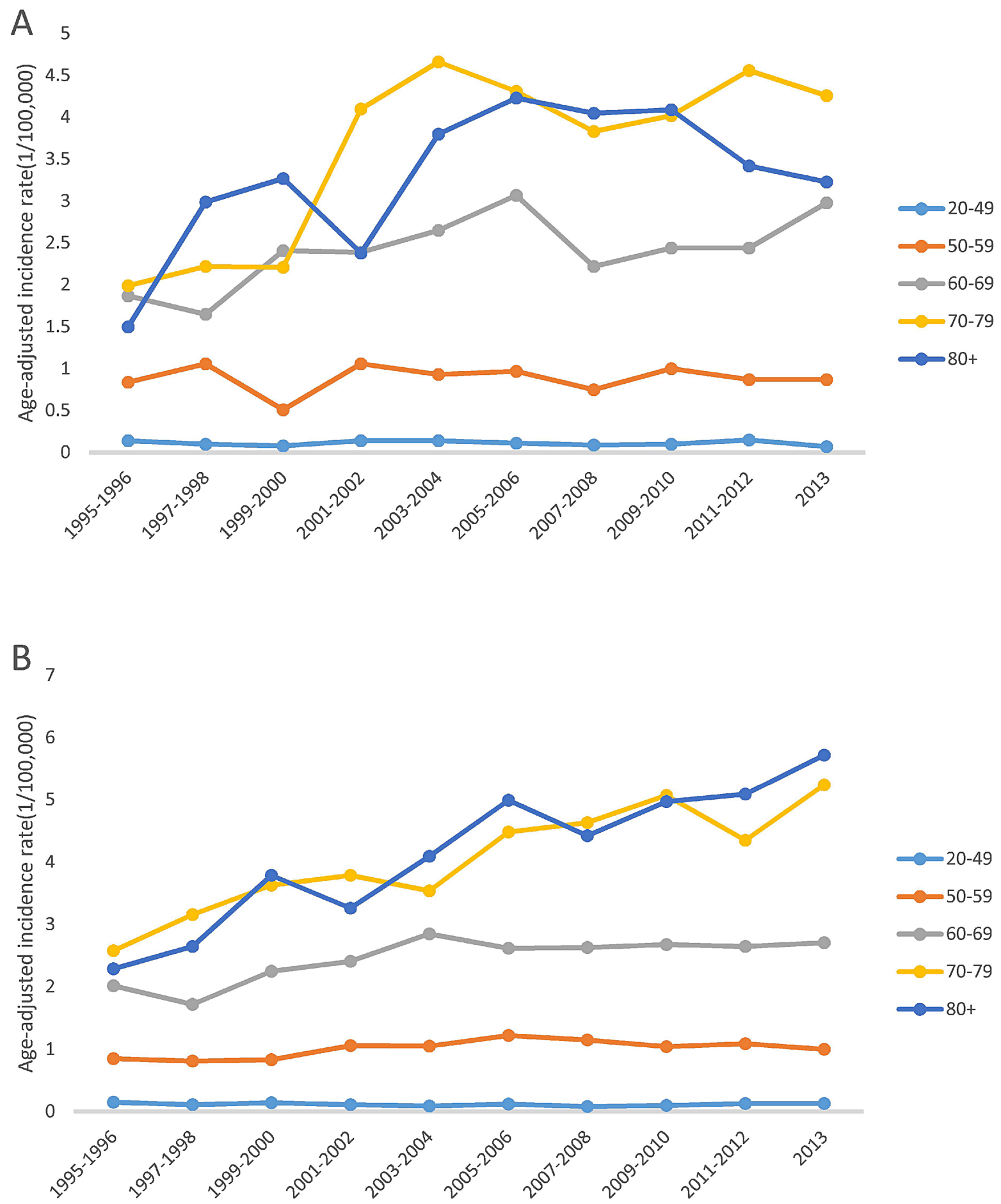

Figure 4: MCL age-adjusted incidence trends by age group, 1995-2013. (A) Texas. (B) SEER. 
(Texas and 13 SEER areas). The incidence rates of MCL cancer were compared among those four time periods and by geographic areas.

\section{Main outcome variables}

The primary outcome variable was cumulative MCL incidence rate in Texas and SEER areas from 1995 to 2013. IRs were expressed as number of new cases per 100,000 persons per year in a certain time period and were standardized to the 2000 U.S. population.

\section{Other variables}

Covariates included age at diagnosis, gender, race/ ethnicity, and tumor stage. Age was classified according to five categories with $<50,50-59,60-69,70-79$ and $\geq 80$ years. Gender was a binary variable with male and female. Race/ethnicity was categorized as non-Hispanic white, non-Hispanic black, Hispanics, and other. Lymphoma stage was classified as localized, regional, advanced (Ann Arbor stages 3-4, distant stage recorded in SEER) and unknown stages.

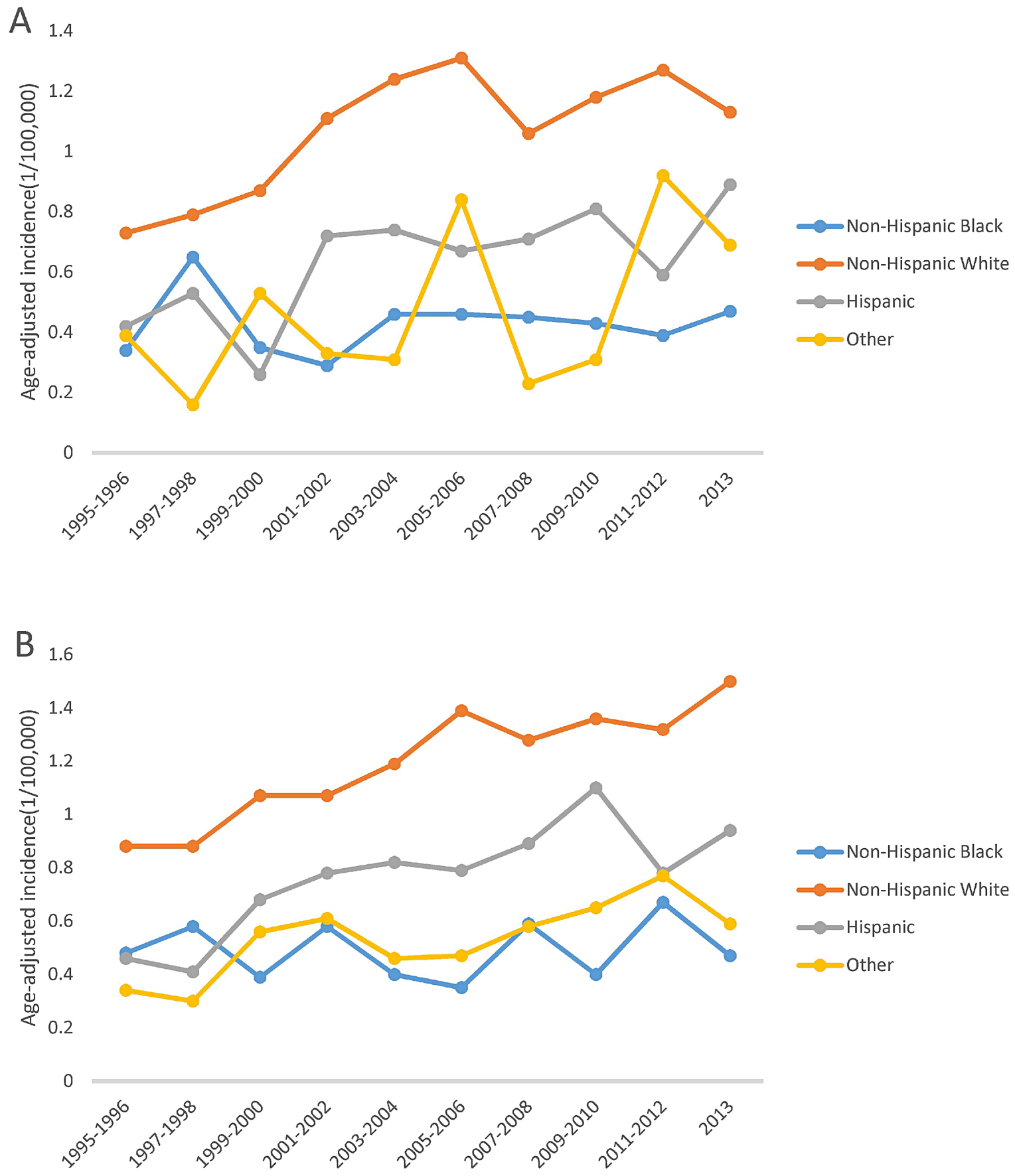

Figure 5: MCL age-adjusted incidence trends by race/ethnicity, 1995-2013. (A) Texas. (B) SEER. 


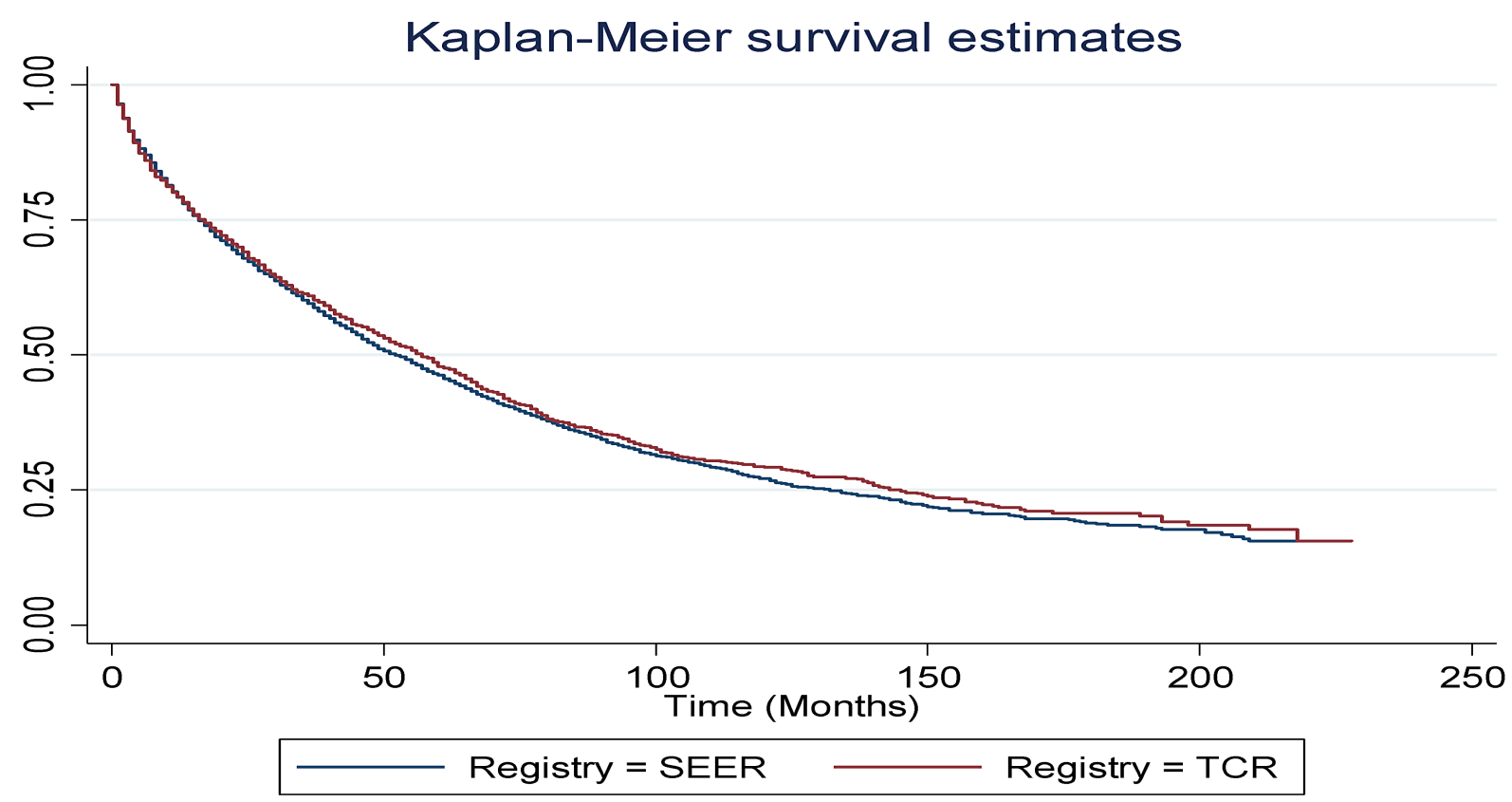

Figure 6: Kaplan-Meier survival estimates for SEER and TCR.

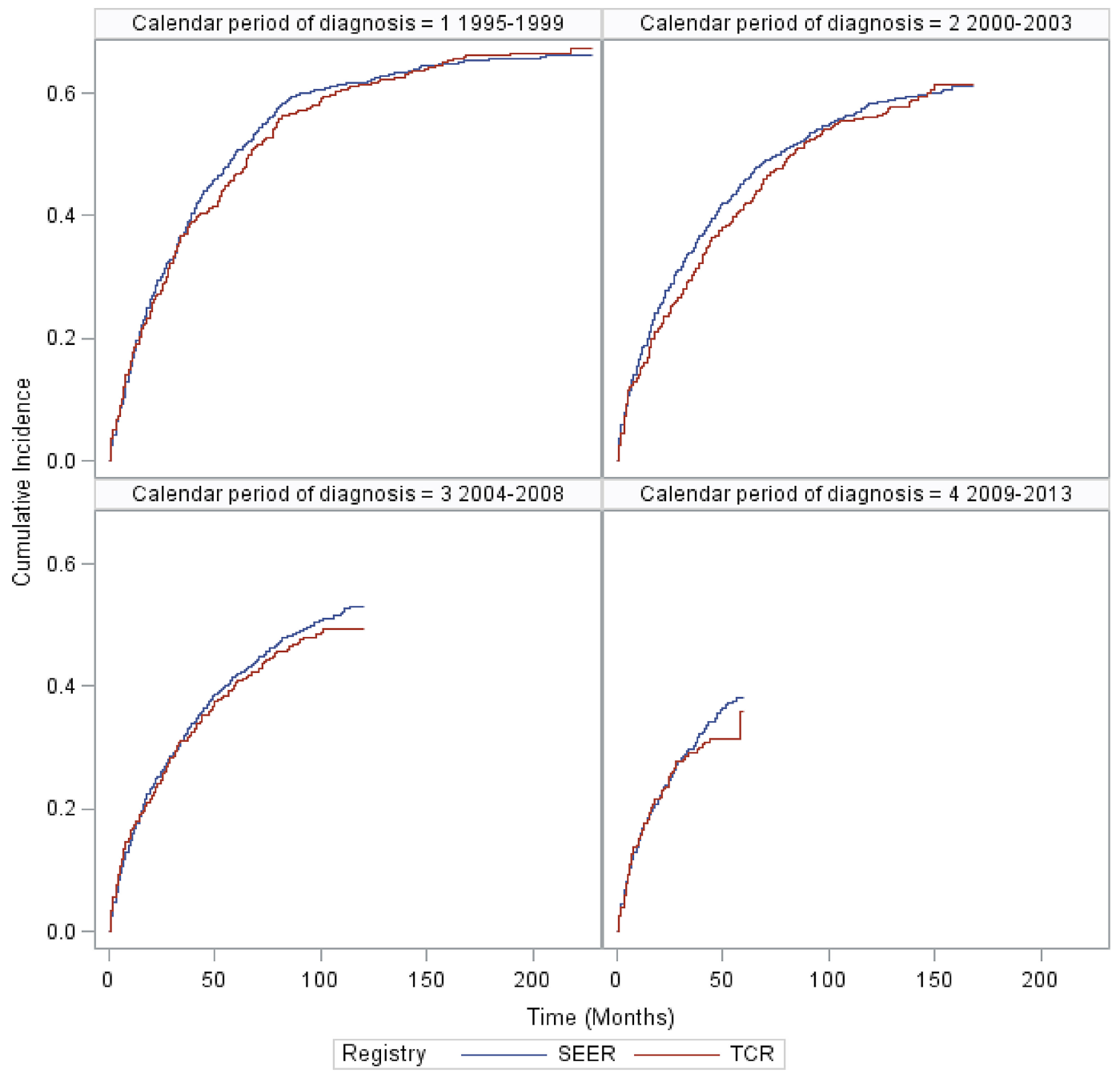

Figure 7: Cumulative incidence of MCL death for SEER and TCR areas over calendar period of diagnosis. 


\section{Statistical analysis}

Relative risk was expressed as the ratio of incidence rates between two comparison groups. Incidence rates were stratified by age group, gender, race/ethnicity, tumor stage and region (Texas vs. SEER areas) and were plotted against diagnostic periods. Annual percent changes in incidence rates were also calculated.

We used negative binomial regression model for count data to assess the association between the year of diagnosis and MCL incidence rates. The negative binomial regression model is an alternative to Poisson regression model when over-dispersion existed in the data. The count data was stratified by diagnostic period, age group, gender, race/ ethnicity and tumor stages. Population at risk for each group during each time period was calculated using SEER ${ }^{*}$ stat 8.3.4 and was used as an offset in the negative binomial regression after a log transformation [27]. Negative binomial regression was conducted using SAS enterprise guide 7.1. All regression models included age, gender, race/ethnicity and tumor stage as covariates. Subgroup incidence and trend analyses were conducted for high risk groups.

Overall survival for MCL patients in SEER and TCR areas was analyzed and plotted. Cumulative incidence function was also used to calculate the cumulative incidence of death for MCL patients. Cumulative incidence of death for MCL patients was plotted by calendar period of diagnosis and tumor stage.

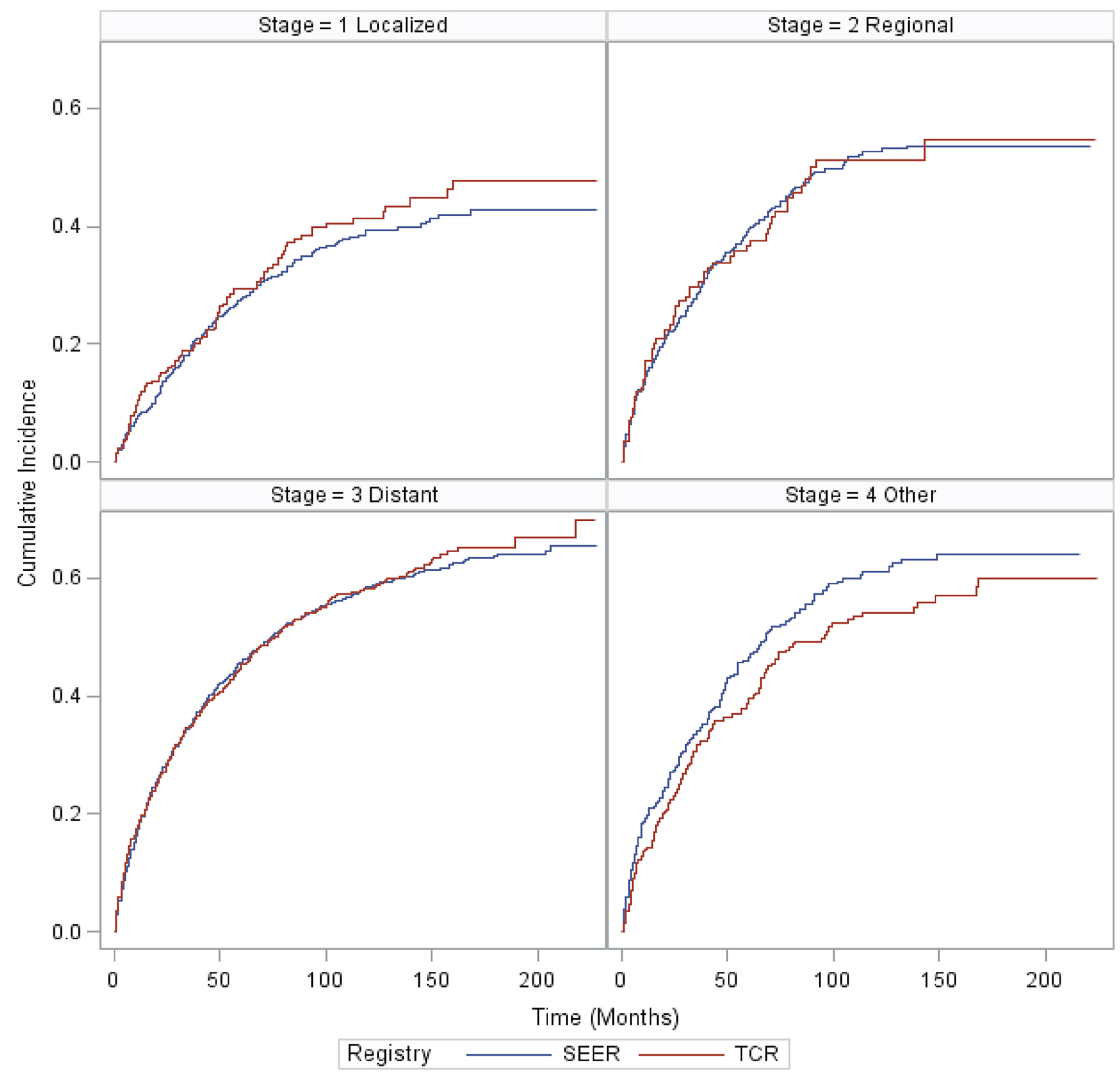

Figure 8: Cumulative incidence of MCL death for SEER and TCR areas by tumor stage. 


\section{CONCLUSIONS}

From 1995 to 2013, MCL incidence rates were significantly higher in male, non-Hispanic white, elderly patients with advanced stage tumors in both Texas and SEER areas. MCL incidence rates also increased over time in both Texas and SEER areas, with increases being greater in male, white, elderly patients (aged $\geq 70$ years) with advanced stage tumors. When comparing Texas to SEER areas, we found that the increase of MCL incidence rates has slowed down for the past few years, especially in male and patients with advanced stage tumors. In general, Texas has similar MCL incidence trends and disparities as SEER areas, but the incidence rates in Texas were slightly lower than that in SEER areas. Future etiology studies, cancer prevention, and treatment development programs should focus on those high risk populations in both Texas and SEER areas.

\section{Abbreviations}

NHL, non-Hodgkin lymphoma; MCL, mantle cell lymphoma; SEER, Surveillance, Epidemiology and End Results; TCR, Texas Cancer Registry; IR, Incidence rates; RR, Relative risk; APC, annual percent change; ICD-O-3, International Classification of Diseases for Oncology 3rd edition; R-CHOP, rituximab plus cyclophosphamide, doxorubicin, vincristine, and prednisone regimen.

\section{Author contributions}

SF: Study concept and design; data collection, analysis, and interpretation; manuscript drafting and revision; MW: Study concept and design; data interpretation; manuscript drafting and revision; DL: Study concept and design; data interpretation; manuscript drafting and revision; RL: Study concept and design; data interpretation; manuscript drafting and revision; BZ: Study concept and design; data collection, analysis, and interpretation; XLD: Study concept and design; data interpretation; manuscript drafting and revision.

\section{ACKNOWLEDGMENTS}

We acknowledge the efforts of the Texas Cancer Registry and the National Cancer Institute in the creation of these databases. The interpretation and reporting of these data are the sole responsibilities of the authors.

\section{CONFLICTS OF INTEREST}

None.

\section{FUNDING}

None.

\section{REFERENCES}

1. Cancer Stat Facts: Non-Hodgkin Lymphoma. Surveillance, Epidemiology, and End Results (SEER) Program of the National Cancer Institute. 2017. Available from: https://seer. cancer.gov/statfacts/html/nhl.html.

2. A clinical evaluation of the International Lymphoma Study Group classification of non-Hodgkin's lymphoma. The Non-Hodgkin's Lymphoma Classification Project. Blood. 1997; 89:3909-18.

3. Zhou Y, Wang H, Fang W, Romaguer JE, Zhang Y, Delasalle KB, Kwak L, Yi Q, Du XL, Wang M. Incidence trends of mantle cell lymphoma in the United States between 1992 and 2004. Cancer. 2008; 113:791-8.

4. Swerdlow SH, Campo E, Harris NL, Jaffe ES, Pileri SA, Stein $\mathrm{H}$, Thiele J, Vardiman JW. WHO Classification of Tumours of Haematopoietic and Lymphoid Tissues. Fourth ed. Lyon, France: International Agency for Research on Cancer; 2008.

5. Vose JM. Mantle cell lymphoma: 2015 update on diagnosis, risk-stratification, and clinical management. Am J Hematol. 2015; 90:739-45.

6. Aschebrook-Kilfoy B, Caces DB, Ollberding NJ, Smith $\mathrm{SM}$, Chiu BC. An upward trend in the age-specific incidence patterns for mantle cell lymphoma in the USA. Leuk Lymphoma. 2013; 54:1677-83.

7. Banks PM, Chan J, Cleary ML, Delsol G, De Wolf-Peeters C, Gatter K, Grogan TM, Harris NL, Isaacson PG, Jaffe ES. Mantle cell lymphoma. A proposal for unification of morphologic, immunologic, and molecular data. Am J Surg Pathol. 1992; 16:637-40.

8. Wang Y, Ma S. Racial differences in mantle cell lymphoma in the United States. BMC Cancer. 2014; 14:764.

9. Lee PG, Cigolle C, Blaum C. The co-occurrence of chronic diseases and geriatric syndromes: the health and retirement study. J Am Geriatr Soc. 2009; 57:511-6.

10. Vogeli C, Shields AE, Lee TA, Gibson TB, Marder WD, Weiss KB, Blumenthal D. Multiple chronic conditions: prevalence, health consequences, and implications for quality, care management, and costs. J Gen Intern Med. 2007; 3:391-5.

11. Herrera AP, Snipes SA, King DW, Torres-Vigil I, Goldberg DS, Weinberg AD. Disparate inclusion of older adults in clinical trials: priorities and opportunities for policy and practice change. Am J Public Health. 2010; 100:S105-12.

12. Cheah CY, Seymour JF, Wang ML. Mantle Cell Lymphoma. J Clin Oncol. 2016; 34:1256-69.

13. Eng A, 't Mannetje A, McLean D, Ellison-Loschmann L, Cheng S, Pearce N. Gender differences in occupational exposure patterns. Occup Environ Med. 2011; 68:888-94.

14. Campos-Serna J, Ronda-Perez E, Artazcoz L, Moen BE, Benavides FG. Gender inequalities in occupational health related to the unequal distribution of working and employment conditions: a systematic review. Int J Equity Health. 2013; 12:57. 
15. Jares P, Colomer D, Campo E. Molecular pathogenesis of mantle cell lymphoma. J Clin Invest. 2012; 122:3416-23.

16. Hirt C, Schuler F, Dolken L, Schmidt CA, Dolken G. Low prevalence of circulating $\mathrm{t}(11 ; 14)(\mathrm{q} 13 ; \mathrm{q} 32)$-positive cells in the peripheral blood of healthy individuals as detected by real-time quantitative PCR. Blood. 2004; 104:904-5.

17. Leroux D, Le Marc'Hadour F, Gressin R, Jacob MC, Keddari E, Monteil M, Caillot P, Jalbert P, Sotto JJ. NonHodgkin's lymphomas with $\mathrm{t}(11 ; 14)(\mathrm{q} 13 ; \mathrm{q} 32)$ : a subset of mantle zone/intermediate lymphocytic lymphoma? Br J Haematol. 1991; 77:346-53.

18. Fisher RI, Bernstein SH, Kahl BS, Djulbegovic B, Robertson MJ, de Vos S, Epner E, Krishnan A, Leonard JP, Lonial S, Stadtmauer EA, O'Connor OA, Shi H, et al. Multicenter phase II study of bortezomib in patients with relapsed or refractory mantle cell lymphoma. J Clin Oncol. 2006; 24:4867-74.

19. Flinn IW, van der Jagt R, Kahl BS, Wood P, Hawkins TE, Macdonald D, Hertzberg M, Kwan YL, Simpson D, Craig M, Kolibaba K, Issa S, Clementi R, et al. Randomized trial of bendamustine-rituximab or R-CHOP/R-CVP in first-line treatment of indolent NHL or MCL: the BRIGHT study. Blood. 2014; 123:2944-52.

20. Wang M, Fayad L, Wagner-Bartak N, Zhang L, Hagemeister F, Neelapu SS, Samaniego F, McLaughlin P, Fanale M, Younes A, Cabanillas F, Fowler N, Newberry KJ, et al. Lenalidomide in combination with rituximab for patients with relapsed or refractory mantle-cell lymphoma: a phase 1/2 clinical trial. Lancet Oncol. 2012; 13:716-23.

21. Wang ML, Rule S, Martin P, Goy A, Auer R, Kahl BS, Jurczak W, Advani RH, Romaguera JE, Williams ME, Barrientos JC, Chmielowska E, Radford J, et al. Targeting BTK with ibrutinib in relapsed or refractory mantle-cell lymphoma. N Engl J Med. 2013; 369:507-16.
22. Hess G, Herbrecht R, Romaguera J, Verhoef G, Crump M, Gisselbrecht C, Laurell A, Offner F, Strahs A, Berkenblit A, Hanushevsky O, Clancy J, Hewes B, et al. Phase III study to evaluate temsirolimus compared with investigator's choice therapy for the treatment of relapsed or refractory mantle cell lymphoma. J Clin Oncol. 2009; 27:3822-9.

23. Geisler CH, Kolstad A, Laurell A, Jerkeman M, Raty R, Andersen NS, Pedersen LB, Eriksson M, Nordstrom M, Kimby E, Bentzen H, Kuittinen O, Lauritzsen GF, et al. Nordic MCL2 trial update: six-year follow-up after intensive immunochemotherapy for untreated mantle cell lymphoma followed by BEAM or BEAC + autologous stem-cell support: still very long survival but late relapses do occur. Br J Haematol. 2012; 158:355-62.

24. Bernstein SH, Epner E, Unger JM, Leblanc M, Cebula E, Burack R, Rimsza L, Miller TP, Fisher RI. A phase II multicenter trial of hyperCVAD MTX/Ara-C and rituximab in patients with previously untreated mantle cell lymphoma; SWOG 0213. Ann Oncol. 2013; 24:1587-93.

25. QuickFacts Texas. United States Census Bureau; 2015 [cited July 19, 2016]. Available from: http://www.census. gov/quickfacts/table/PST045215/48.

26. Chapter II: Historical background and status of North American standards-Surveillance Epidemiology and End Results Program. North American Association of Central Cancer Registries [cited 10/24/2016]. Available from: https:/www.naaccr.org/Applications/ContentReader/ Archive/13/Chap02.html.

27. Cameron AC, Trivedi PK. Regression Analysis of Count Data. Second ed. New York: Cambridge Press; 1998. 